\title{
Alternative Proof of Sine's Theorem on the Size of a Regular Polygon in $\mathbf{R}^{n}$ with the $\ell_{\infty}$-Metric
}

\author{
A. Blokhuis and H. A. Wilbrink \\ Department of Mathematics and Computing Science, Eindhoven University of Technology, \\ Den Dolech 2, The Netherlands
}

\begin{abstract}
It is shown that a regular polygon in $\mathbf{R}^{n}$ with the $\ell_{\infty}$-metric has at most $(2 n)^{n}$ vertices.
\end{abstract}

\section{Introduction}

Let $\mathbf{R}^{n}$ be equipped with the $\ell_{\infty}$-metric, i.e., $d_{\infty}(\mathbf{x}, \mathbf{y})=\max _{1 \leq i \leq n}\left|x_{i}-y_{i}\right|$. A cyclically ordered set $\mathbf{p}_{0}, \mathbf{p}_{1}, \ldots, \mathbf{p}_{m-1}$ of points in $\mathbf{R}^{n}$ is called a regular $m$-gon if $d\left(\mathbf{p}_{i}, \mathbf{p}_{j}\right)=d\left(\mathbf{p}_{i+k}, \mathbf{p}_{j+k}\right)$ for all $i, j, k \in\{0, \ldots, m-1\}$ (where indices are computed modulo $m$ ).

Nussbaum [2] has asked the question: for which $m$ does there exist a regular $m$-gon in $\mathbf{R}^{n}$ (with the $\ell_{\infty}$-metric)? He has shown that necessarily $m \leq f(n)$ for some rapidly growing function of $n$, but he asks whether $f(n)=2^{n}$ is the proper bound. We obtain the same result for $f(n)=(2 n)^{n}$. In fact we prove the following: Let $(A, d)$ be a (finite) metric space with a transitive automorphism group of isometries acting on $A$ and suppose that $(A, d)$ is isometrically embeddable in $\left(\mathbf{R}^{n}, d_{\infty}\right)$. Then $A$ has at most $(2 n)^{n}$ points. Note that this result is essentially of the right order of magnitude since there is a finite metric space $A$ of size $2^{n} \cdot n$ ! with transitive group $2^{n} \cdot \operatorname{Sym}(n)$ that can be embedded, namely all points of the form $( \pm 1, \pm 2, \ldots, \pm n)$ with coordinates permuted in all possible ways. However, it is still not known whether, in the more restrictive case that $A$ is a regular $m$-gon, $A$ contains at most $2^{n}$ points.

\section{The Result}

Theorem. Let $(A, d)$ be a (finite) metric space with a transitive group of isometries and suppose that $(A, d)$ is isometrically embeddable in $\left(\mathbf{R}^{n}, d_{\infty}\right)$. Then $A$ has at most $(2 n)^{n}$ points. 
Proof. Let $(A, d)$ be a metric space, with transitive automorphism group $G$, isometrically embedded in $\left(\mathbf{R}^{n}, d_{\infty}\right)$ (note that we do not require $G$ to act on $\left(\mathbf{R}^{n}, d_{\infty}\right)$ ). We define a partial order $\leq_{i}$ on $A$ by $\mathbf{p} \leq_{i} \mathbf{q}$ iff $d(\mathbf{p}, \mathbf{q})=q_{i}-p_{i}$ (note that the relation $\leq_{i}$ is indeed transitive). Let $r_{i}$ be the length of the longest $\leq_{i}$-chain and put $r=\max _{i} r_{i}$. Let $\mathbf{p} \in A$. Define the $i$-height of $\mathbf{p}$ to be the length of the longest descending $i$-chain starting with p. Finally let the height-vector of $\mathbf{p}$ be the vector whose $i$ th coordinate is the $i$-height of $\mathbf{p}$.

Claim. Different vectors in $A$ have different height-vectors.

Proof. For $\mathbf{p}, \mathbf{q} \in A$ we have that, for some $i, d(\mathbf{p}, \mathbf{q})=\left|p_{i}-q_{i}\right|$, i.e., either $\mathbf{p} \leq_{i} \mathbf{q}$ or $\mathbf{q} \leq_{i} \mathbf{p}$. It follows that $\mathbf{p}$ and $\mathbf{q}$ have different $i$-heights. Since the height-vector has entries between 0 and $r$ we immediately get $|A| \leq(r+1)^{n}$. We proceed to show that $r<2 n$. Choose $i$ with $r=r_{i}$ and fix a maximal $\leq_{i}$-chain $\mathbf{p}^{0} \leq_{i}$ $\mathbf{p}^{1} \leq_{i} \cdots \mathbf{p}^{\prime}$. Let $\mathbf{p}$ be an arbitrary point and take $g \in G$ with $\mathbf{p}=g\left(\mathbf{p}^{k}\right)$. Such a $g$ exists since $G$ is transitive on $A$. It follows that, for some $j, g\left(\mathbf{p}^{0}\right), g\left(\mathbf{p}^{1}\right), \ldots, g\left(\mathbf{p}^{r}\right)$ is a $\leq_{j}$-chain. Indeed, for some $j, d\left(g\left(\mathbf{p}^{0}\right), g\left(\mathbf{p}^{r}\right)=\left|p_{j}^{0}-p_{j}^{r}\right|\right.$ and, for each $l, 0<l<r$, we have that $d\left(g\left(\mathbf{p}^{0}\right), g\left(\mathbf{p}^{r}\right)\right)=d\left(g\left(\mathbf{p}^{0}\right), g\left(\mathbf{p}^{l}\right)\right)+d\left(g\left(\mathbf{p}^{l}\right), g\left(\mathbf{p}^{r}\right)\right)$. From this it follows that $\mathbf{p}$ has $j$-height $k$ (if $g\left(\mathbf{p}^{0}\right) \leq{ }_{j} g\left(\mathbf{p}^{r}\right)$ ) or $r-k$ (if $\left.g\left(\mathbf{p}^{r}\right) \leq_{j} g\left(\mathbf{p}^{0}\right)\right)$. So of every pair $\{k, r-k\}(0 \leq k \leq r / 2)$ at least one element occurs somewhere in the weight-vector of $\mathbf{p}$. Since the weight-vector has only $n$ coordinates we get $r<2 n$.

Note that we prove slightly more, in fact, in the "extremal" case, i.e., the case $r=2 n-1$ we get that the number of possible height-vectors is precisely $2^{n} \cdot n$ !, which is best possible but we do not see how to improve the general case further, $0<r<2 n$.

\section{References}

1. M. A. Akoglu and U. Krengel, Nonlinear models of diffusion on a finite space, Probability Theory and Relative Fields $76(1987), 411-420$.

2. Roger D. Nussbaum, Omega limit sets of nonexpansive maps: finiteness and cardinality estimates, Differential and Integral Equations 3 (1990), 523-540.

3. R. C. Sine, On nonlinear contraction semigroups in sup norm spaces, Nonlinear Analysis 3 (1979), $885-890$.

Received June 4, 1990.

Note added in proof. It was pointed out to the authors by Professor Krengel that Nussbaum's question goes back to Sine, and that similar results have been obtained by Shih Kung Loo and Peter Martus. 